\title{
Silicon Adsorption In Single Walled Nanotubes
}

\author{
M. L. Colussi*, L.P. Neves ${ }^{+}$, and R.J. Baierle* \\ ${ }^{* 1}$ Departamento de Física da UFSM, Faixa de Camobi Km 9, 97105-900, Santa Maria-RS, Brazil \\ +Centro Universitário Franciscano, Rua dos Andradas 1614, 97010-032, Santa Maria - RS, Brazil
}

Received on 8 December, 2005

\begin{abstract}
Using density functional (DF) calculations and Monte Carlo (MC) simulations we have investigated the main electronic and structural properties of silicon interacting with single walled carbon nanotubes. We have investigated the silicon adsorption externally and internally in the nanotubes surface. The total energies calculations and charge density plot present that the adsorption is most stable externally with silicon forming four bonds with the $\mathrm{C}$ atoms and the $\mathrm{Si}-\mathrm{C}$ bond distances are similar to the ones in the $\mathrm{SiC}$ crystal. When silicon is adsorbed in a semiconductor nanotube, a new occupied electronic level inside the band gap is observed. For the metallic tube, the electronic silicon levels are close to the Fermi energy, increasing the metallic tube character.
\end{abstract}

Keywords: Nanotube; Silicon adsorption; Monte Carlo simulations; First principle calculation

\section{INTRODUCTION}

Carbon nanotubes (CNTs) were identified for the first time by S. Iijima in 1991 as by-products of arch discharge experiments[1]. They can be considered a new form of matter and are some of the first true nanomaterials engineered at the molecular level. They exhibit unique structural and electronic properties and can be considered quasi-one-dimensional materials, with promising applications in nanometric devices[2]. The electronic and structural properties of single walled carbon nanotubes (SWCNTs) have been theoretically studied [3] and confirmed experimentally [4]. The main electronic features can be understand using the hexagonal graphite network as guide[2]. Tubular graphitic forms of $\mathrm{Si}$ and Ge have been proposed [5], but no stable forms have yet being obtained. The difficulties for $\mathrm{Si}$ and $\mathrm{Ge}$ form hexagonal graphitic network can be associated with the considerable energies difference between the $\mathrm{sp}^{2}$ and the $\mathrm{sp}^{3}$ hybridization[6].

Silicon is the most important material in the microelectronics and the interaction between nanotubes and Si is a very important subject that need to be clarified. The substitutional silicon doping in SWCNTs has been theoretically studied[7]. The formation energies are around $3.1 \mathrm{eV}$ and the main electronic feature is an unoccupied level inside the band gap for a semiconductor nanotube. The interaction between a tube and the clean $\mathrm{Si}(001)$ surface have been recently studied by Orellana et al.[8]. They have shown that the nanotube adsorption (contact) by the silicon surface increases the density of states near the Fermi level, enhancing the metallic character of the nanotube.

In this work, using Monte Carlo (MC) simulations with a many-body empirical potential and first principle calculations, we have studied the adsorption of a $\mathrm{Si}$ atom internally and externally in two different nanotubes: the semiconductor zigzag $(10,0)$ and the metallic armchair $(6,6)$. In the next section we present the methodology used, in section three the results and discussion, and in section four our conclusions.

\section{METHODOLOGY}

Using empirical many-body potential with the Monte Carlo method [9], the binding energy is

$$
E_{\text {bind }}=\sum_{i} \sum_{j>i}\left[V_{R}\left(r_{i j}\right)-B_{i j} V_{A}\left(r_{i j}\right)\right]
$$

where $\mathrm{r}_{i j}$ is the scalar distance between atoms $\mathrm{i}$ and $\mathrm{j}, \mathrm{V}_{R}\left(\mathrm{r}_{i j}\right)$ and $\mathrm{V}_{A}\left(\mathrm{r}_{i j}\right)$ represent a pair-additive core-core repulsion and an attraction due to the valence-electrons, respectively. $\mathrm{B}_{i j}$ is a many-body empirical bond order that couples quantities such as bond angles and local coordination to the attractive potential. Tersoff $[10,11]$ has shown that, if this function is used for the pairs terms, a wide range of structural properties of materials, including carbon and silicon, can be appropriately describing using this formalism, providing a reasonable starting point for predicting trends, such as those used here. This empirical potential was a fit to the lattice constant and binding energy of a number of carbon (or silicon) lattices as well as the elastic constants and vacancy formation energies of graphite (or diamond). Robertson et al. [12] has used this potential to study the energetic and elastic properties of carbon nanotubes and Hamada et al. [13] to generate tube structures, subsequently used in tight-binding electronic structure calculations. Recently, Fagan et. al. [14] have used this potential to study the stability and thermal behavior of silicon nanotubes.

First principle calculations were performed using density functional theory (DFT) with the local spin density approximation (LSDA) for the exchange-correlation term [15] as proposed by Perdew-Zunger[16]. We have used the SIESTAcode [17], which performs a fully self-consistent calculation by solving the standard Kohn-Shan (KS) equations. The KS orbital are represented by a linear combination of pseudoatomic numerical orbital as proposed by Sankey and Niklewski [18]. The ion-electron interaction is modulated by ab initio norm-conserving fully separable TroullierMartins[19] pseudopotentials in the Kleinman-Bylander form [20]. A double-zeta enhanced by diffuse functions (DZP) quality basis set is employed in all calculations. The charge density was projected on a real space grid with an equivalent 
cutoff of 80 Ry to calculate the self consistent Hamiltonian matrix elements. To sampling the Brillouin zone (BZ) a set of three Morkhost-Pack special k-points [21], along the tube axis, has been used.

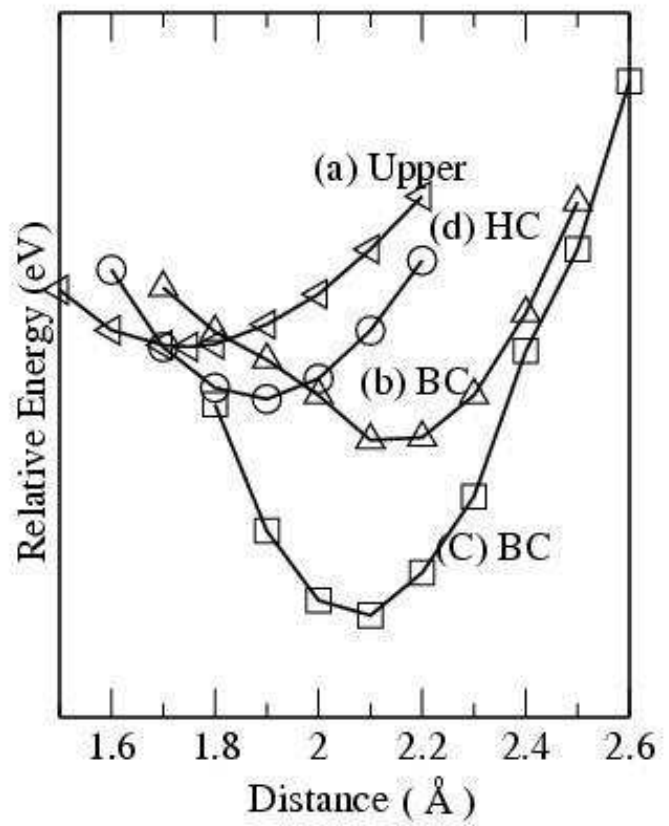

FIG. 1: Relative Energy for Si atom on a external surface in a $(10,0)$ nanotube. In axis $\mathrm{X}$ the distances are in Angstroms and represent the $\mathrm{Si}-\mathrm{C}$ distance when (a) the $\mathrm{Si}$ is adsorbed on the top of a $\mathrm{C}$ atom, (b-c) in the bond center for the two C-C possible bonds orientation (parallel or making $60^{\circ}$ with the tube axis) and (d) the distance from the $\mathrm{Si}$ to a hexagon center.

Our study is performed using two tubes with different chiralities: the semiconductor $(10,0)$ zigzag with $9.924 \AA$ of diameter and the metallic $(6,6)$ armchair with $10.312 \AA$ of diameter. We use periodic boundary conditions with a tetragonal supercell with dimensions of $16 \AA$ in directions perpendicular to the tube axis and the translational symmetry along the tube axis. This construction should eliminated possible intertube interaction or inter adsorbed silicon atoms. The supercell for the $(10,0)$ tube have $120 \mathrm{C}$ atoms and for the $(6,6)$ have 72 atoms, in each case we are using three conventional unit cells. All the atoms in the enlarged unit cell are relaxed. The forces are calculated using the Hellmann-Feymann procedure and the geometry are optimized using the Conjugated Gradient scheme (CG). The system is relaxed until the root mean square criterion of $0.05 \mathrm{eV} / \AA$ on the atomic force is reached.

In the first principle calculations, the binding energies of a given system are determined using total energies calculations, according to equation:

$$
E_{b}=E_{t}[t u b e+S i]-E_{t}[t u b e]-E_{t}[S i]
$$

where $\mathrm{E}_{b}$ and $\mathrm{E}_{t}$ are the formation and total energies, respectively. Negatives values for the binding energy means that the adsorption is exothermic.

\section{RESULTS AND DISCUSSION}

Carbon nanotubes (CNTs) exhibit unique structural and electronic properties. For example zigzag $(n, 0)$, with $n$ not being a multiple of 3 , are semiconductor with a finite gap that depend only the tube diameter while the armchair $(n, n)$ present a metallic behavior.

The gap and the density of states around the Fermi level for the CNTs are predicted to be dependent on the chemi$\mathrm{cal} /$ physical adsorption of atoms and molecules by the tube wall. In this work we have studied the main electronic and structural properties when a silicon atom is adsorbed externally and internally in SWCNTs.

Our empirical calculations are not able to detect energy differences between different tubes. For the two tubes studied we obtain that the cohesion energy per atom is $7.25 \mathrm{eV}$, these value is close to the cohesion energy per atom in the graphitic structure $(7.4 \mathrm{eV})$ obtained using the same approach $[10,11]$.

When silicon is adsorbed on the tube wall, we observed a preference for the external surface. For the $(6,6)$ armchair, the binding energies are $-1.51 \mathrm{eV}$ and $-0.47 \mathrm{eV}$ for the external and internal surface, respectively. For the $(10,0)$ zigzag these values are $-1.41 \mathrm{eV}$ and $-0.32 \mathrm{eV}$.

In our ab initio calculations we studied the $\mathrm{Si}$ adsorption in three candidate sites: center of hexagon, on the top a $\mathrm{C}$ atom and in the bond center. Fig. 1 show the results obtained when the $\mathrm{Si}$ atom is approximated to the external tube surface in the $(10,0)$ semiconductor tube, for the three candidates sites.

The most stable position, lower binding energy, for the three sites investigated is the bond center position where the silicon atom is connected with the $\mathrm{C}$ atoms by four bonds, as indicated in the Fig. 2. Results for the armchair and the internal surface are similar. The calculated binding energy are $-2.80 \mathrm{eV}$ (external) and -2.46 eV (internal) and -2.61 (external) and $-2.22 \mathrm{eV}$ (internal) for the $(10,0)$ and the $(6,6)$ nanotubes, respectively.

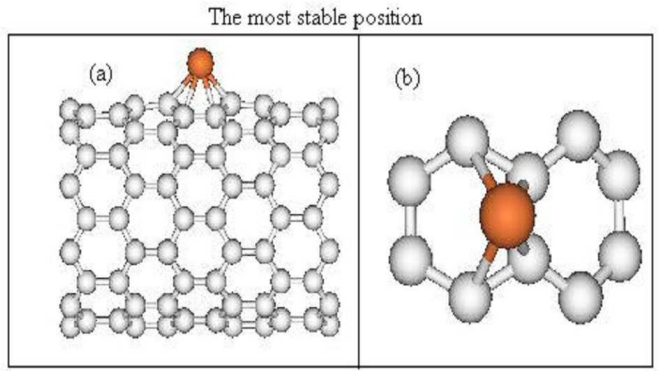

FIG. 2: Silicon adsorbed on the external surface in a $(10,0)$ semiconductor nanotube. Part (a) is a lateral sight and part (b) is the top sight. The Si-C bond distance are $2.1 \AA$.

Similar to the substitutional case [7], new electronic levels near the Fermi level can be observed. In Fig. 3 we present the bands structure obtained when a $\mathrm{Si}$ atom is adsorbed externally and internally for the two nanotubes studied, the $(10,0)$ (a-c) and $(6,6)(d-e)$, respectively. 
(a)

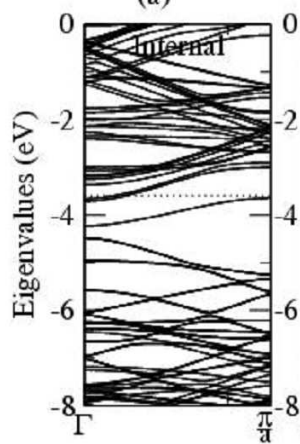

(d)

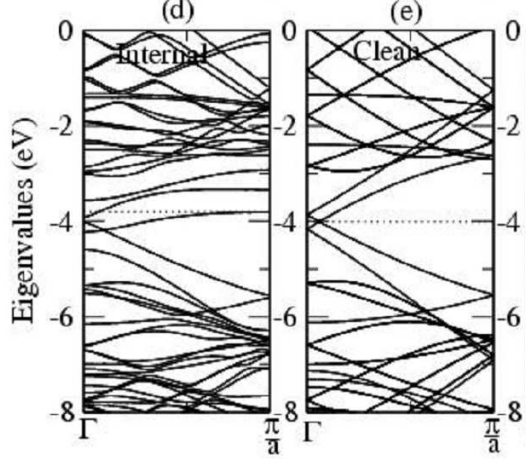

(c)

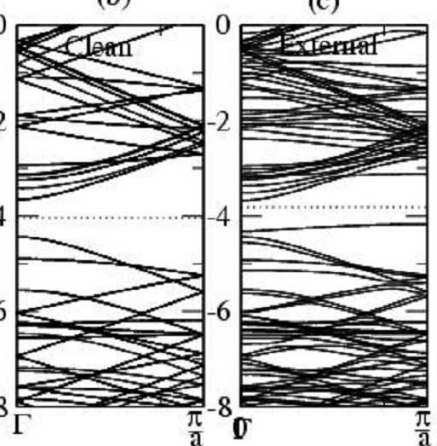

(f)

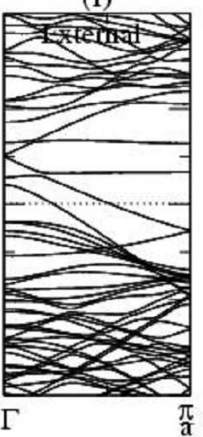

FIG. 3: Bands Structure for the $(10,0)(\mathrm{a}-\mathrm{c})$, and $(6,6)(\mathrm{d}-\mathrm{f})$ nanotubes when a Si atom is adsorbed in the tube wall. In parts (a) and (d) the $\mathrm{Si}$ is bonded on the externally and in parts (c) and (f) internally. Parts (b) and (e) are the bands structure for the "clean" surface. The dotted lines indicate the Fermi energy.

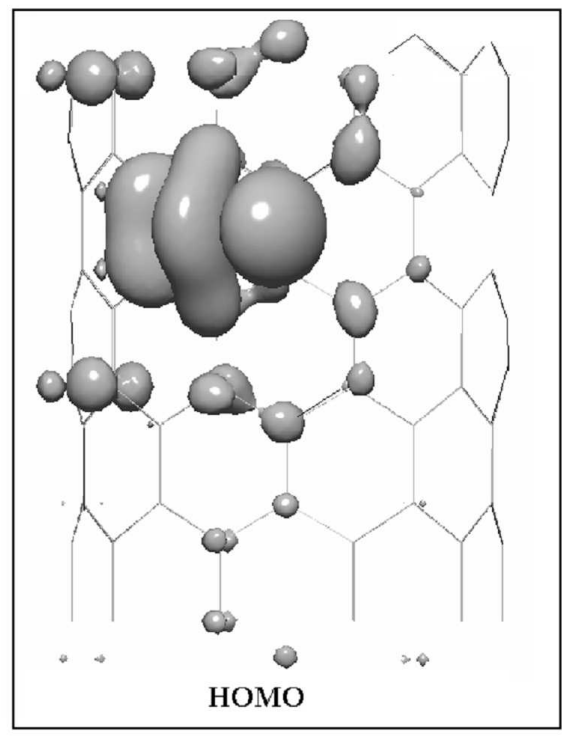

FIG. 4: Spatial localization of the charge density for the HOMO when a silicon atom is adsorbed in the external surface of a $(10,0)$ carbon nanotube.
For the semiconductor $(10,0)$ the appearance of the new electronic levels can be more clearly observed. For the externally (Fig.3-c) and internally (Fig 3-a) adsorption an occupied electronic level, the HOMO (highest occupied molecular orbital) is present in the tube gap.

In figure 4 we can observe that the HOMO is localized on the foreign impurity ( $\mathrm{Si}$ atom) and the $\mathrm{C}$ atoms that are bonded with the silicon. A similar charge density plot (not showed here) for the bottom of the conduction band show a weakly contribution from the $\mathrm{Si}$ atom. For the $(6,6)$ nanotube the electronic levels near to the Fermi energy have a strong contribution from the $\mathrm{Si}$ atom, indicating that the adsorbed $\mathrm{Si}$ atom introduce localized states close to the Fermi energy in a metallic nanotube, enhanced the metallic character.

An analysis of the Mülliken population presents a charge transference from the nanotube to the silicon. For the $(10,0)$ tube we obtain $0.176 e$ ( $e$ is the fundamental electric charge) and $0.054 e$, for the internal and external surface, respectively. For the $(6,6)$ tube the charge transferred is $0.024 e$ and 0.194 $e$. The charge transference is in agreement with the binding energy results.

\section{CONCLUSIONS}

In conclusion, our calculations for the binding energy present that $\mathrm{Si}$ is chemically adsorbed on the tube surface with a preference for the external surface (lower binding energy), that is confirmed by the charge density plot and the Mulliken population analysis. When the silicon is adsorbed in a semiconductor $(10,0)$ tube a new occupied electronic level will be present in the gap. For the metallic $(6,6)$ tube we observe an increase in the density of states near the Fermi level, as consequence the tube metallic character is enhanced. The silicon adsorption is an exothermic reaction (negative binding energies) and it can be considered for the possibility to construct tubular forms of mixed SiC compounds[22]. However, the binding energies are lower than the Si cohesive energy indicating that in an Si-rich environment, the $\mathrm{Si}$ atoms should aggregated forming the $\mathrm{Si}$ crystalline (or cluster). When a silicon is substitutional in a SWCNT it is threefold coordinated and for the adsorbed silicon it is fourfold coordinated (in the most stable configuration) making the adsorbed silicon a non candidate for a reactive center. The chemical binding between the $\mathrm{Si}$ adsorbed by the tube surface and a foreign impurity (like $\mathrm{H}, \mathrm{O}, \mathrm{OH}, \mathrm{O}_{2}$ ) must destroy the weak bond between the adsorbed $\mathrm{Si}$ and the tube wall.

\section{Acknowledgement}

This work was supported by Brazilian agencies CAPES and CNPq and FAPERGS. The calculations have been performed using the facilities of the Centro Nacional de Processamento de Alto Desempenho (CENAPAD in Campinas). 
erties of Carbon Nanotubes, London, World Scientific (1998).

[3] R. Saito, M. Fujita, G. Dresselhaus, and M.S. Dresselhaus, Appl. Phys. Lett. 60, 2204 (1992).

[4] S.J. Tans, M.H. Devoret, H. Daí. A. Thess, R. E. Smalley, L.J. Geerligs, and C. Dekker, Nature 386, 474 (1997).

[5] S.B. Fagan., R.J Baierle, R. Mota, A.J.R. da Silva, and A. Fazzio, Phys. Rev. B 61, 9994 (2000); G. Seifert, T. Köhler, H. M. Urbassek, E. Hernandez, and T. Frauenheim, Phys. Rev. B 63, 193409 (2001); G. Seifert, T. Köhler, Z. Hajnal, and T. Frauenheim, Solid State Commun. 119, 653 (2001).

[6] M.T. Yin and M.L. Cohen, Phys. Rev. B 23, 5048 (1981).

[7] R.J. Baierle, S.B. Fagan, R. Mota, A.J.R. da Silva, and A. Fazzio, Phys. Rev. B 64, 085413 (2001).

[8] W. Orellana, R.H. Miwa, and A. Fazzio, Phys. Rev Lett. 91 , 166802, (2003); W. Orrelana, R.H. Miwa, and A. Fazzio, Surf. Science, 566, 728 (2004).

[9] J. M. Hammersley, D.C. Handscomb, Monte Carlo Methods, Wiley, New York, (1979).

[10] J. Tersoff, Phys. Rev. Lett. 56, 632 (1986).

[11] J. Tersoff, Phys. Rev. B 37, 6991 (1998).
[12] D. H. Robertson, D.W. Brenner, J.W. Mintmire, Phys. Rev. B 45, 12592 (1992).

[13] N. Hamada, S. Sawada, A. Oshiyama, Phys. Rev. Lett. 68, 1579 (1992).

[14] S. B. Fagan, R. Mota, R.J. Baierle, G. Paiva, A.J.R. da Silva, and A. Fazzio, J. of Molec. Sturcture 539, 101 (2001).

[15] D. M. Ceperley, B. Alder, J. Phys. Rev. Lett. 45, 566 (1980).

[16] J. P. Perdew, A. Zunger, Phys. Rev. B 23, 5048 (1981).

[17] P. Ordejón, E. Artacho, J. M. Soler, Phys. Rev. B 53, 10441 (1996); D. Sánchez-Portal, P Ordejón,. E.; Artacho, J. M. Soler, Int. J. Quantum Chem. 65, 453 (1997).

[18] O. F. Sankey, D.Nikleswsky, J. Phys. Rev. B 40, 3979 (1989).

[19] N. Toullier, J. L.Martins, Phys. Rev. B 43, 1991 (1993).

[20] L. Kleinman and D.M. Bylander, Phys. Rev. Lett. 48, 1425 (1982).

[21] H. J. Monkhorst, J. D. Pack, Phys. Rev. B 1976, 13, 5188.

[22] X.-H. Sun, C.-P. Li, W.-K. Wong, N.-B. Wong, C.-S. Lee, S.-T. Lee, and B.-K. Teo, J. Am. Chem. Soc. 124, 14464 (2002). 